\section{Análise da implantação da gestão descentralizada em saúde: estudo comparado de cinco casos na Bahia, Brasil}

\author{
The implementation of decentralized health \\ systems: a comparative study of five cases \\ in Bahia, Brazil
}

\author{
${ }^{1}$ Instituto de Saúde Coletiva, \\ Universidade Federal da \\ Bahia, Salvador, Brasil. \\ 2 Escola Nacional de Saúde \\ Pública Sergio Arouca, \\ Fundação Oswaldo Cruz, \\ Rio de Janeiro, Brasil. \\ 3 Groupe de Recherche \\ Interdisciplinaire en Santé, \\ Montreal, Canadá. \\ 4 Département de Médecine \\ Sociale et Préventive, \\ Université de Montreal, \\ Montreal, Canadá. \\ 5 Faculdade de Odontologia \\ Universidade Federal da \\ Bahia, Salvador, Brasil. \\ Correspondência \\ L. M. Vieira-da-Silva \\ Instituto de Saúde \\ Coletiva, Universidade \\ Federal da Bahia. \\ Rua Padre Feijó 29, \\ 4 o andar, Salvador, $B A$ \\ 40110-170, Brasil. \\ ligiamvs@ufba.br
}

\begin{abstract}
Understanding the effects of health decentralization policies in Brazil requires different methodological approaches to capture the issue's complexity from distinct angles. Five case studies were thus performed to evaluate the degree of implementation of components related to decentralization of the health system management in selected municipalities (counties) in Bahia State, Brazil. A logical model was elaborated with definitions related to policy goals. A comparative study of the five municipalities, considered "exemplary cases", showed that decentralization alone does not explain the organizational changes in the municipal health systems. Local government characteristics such as the municipal master plan, governing capacity, and governance proved important for heath care changes. The main problems and insufficiencies were found in the system's management and quality of healthcare delivered to the population. The authors discuss their findings and identify critical areas for future interventions with special emphasis on the institutionalization of planning and evaluation and the development of inter-sector projects.
\end{abstract}

Health System; Health Management; Decentralization
Ligia Maria Vieira-da-Silva ${ }^{1}$ Zulmira M. Araújo Hartz 2,3,4 Sônia Cristina Lima Chaves 5 Gerluce Alves Pontes da Silva ${ }^{1}$ Jairnilson Silva Paim 1

\section{Introdução}

A descentralização de ações governamentais vem sendo implementada em diversos países do mundo com resultados diferenciados 1,2. Problemas nos resultados da descentralização têm sido relacionados com fatores sociais e políticos 3 , particularmente quanto à estratégia 4 e aos conflitos decisórios ${ }^{5}$. Embora haja um consenso sobre os benefícios potenciais do processo de descentralização, os resultados diferenciados e mesmo negativos da sua operacionalização mostram que essa política não é uma panacéia, dependendo da mediação de um conjunto de fatores políticos, sociais e relacionais 6,7 .

No Brasil, a descentralização tem-se constituído em um dos principais componentes do processo de Reforma Sanitária, cujas diretrizes foram aprovadas na 8a Conferência Nacional de

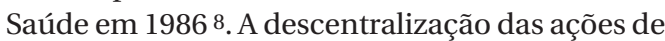
saúde remonta à década de oitenta com as Ações Integradas de Saúde (AIS) e o Sistema Unificado e Descentralizado de Saúde (SUDS), institucionalizando-se e ganhando maior abrangência e densidade a partir da aprovação das suas bases legais $9,10,11,12,13$.

Estudos realizados sobre as repercussões desse processo revelaram, ainda na década de 80, a ampliação da oferta de serviços e da participação popular 14,15. Mais recentemente, estudos de caso sobre a implementação da Norma Operacional Básica (NOB-93) em sete municípios 
em gestão semi-plena, revelaram a ampliação da rede assistencial e a diversificação da oferta de serviço, além de certas medidas relacionadas à reorganização do modelo de atenção 16 .

Após a implantação da Norma Operacional Básica 96 (NOB-96) 12, que assegurou maior autonomia municipal na gestão dos recursos, verificou-se a ampliação da oferta da atenção primária e dos serviços de alta e média complexidade nos 522 municípios que se encontravam na gestão plena do sistema 17. Já em investigação realizada em 45 municípios do Estado do Ceará, não foi observada relação entre a descentralização e a melhoria do desempenho do sistema de saúde 18 .

Estudos de caso em profundidade têm mostrado que não apenas a descentralização é responsável por melhorias na organização do sistema de saúde, mas diversas outras características relacionadas com a gestão podem influenciar as diferenças encontradas 7. Embora esses trabalhos tenham auxiliado na compreensão de alguns aspectos relacionados com a descentralização, várias perguntas ainda precisam ser respondidas. Os efeitos encontrados nesses estudos são robustos? Que outros fatores, além da descentralização, podem influenciar a mudança do modelo assistencial? Quais as suas repercussões no estado de saúde da população?

Tendo em vista as dimensões do Brasil, bem como a complexidade do processo de descentralização envolvendo os três níveis de governo, é necessária a realização de novas investigações que superem as lacunas existentes e contribuam para a identificação de estratégias que permitam a comparabilidade, além de referenciais teóricos capazes de subsidiar a formulação de hipóteses para explicar as diferenças encontradas, particularmente aquelas relacionadas com a organização da atenção. Por esses motivos, a compreensão das repercussões do processo de descentralização sobre a reorganização da atenção à saúde requer a realização de investigações com base em enfoques metodológicos diferenciados, visando a captar a complexidade do objeto a partir de distintos ângulos 15,19,20. Daí a pertinência em articular a análise da implantação com uma avaliação de resultados e com a descrição das características de governo e da gestão capazes de influir no processo de descentralização.

Nesse sentido, o presente estudo tem como objetivos analisar comparativamente a implantação dos componentes relacionados com a descentralização da gestão da saúde em cinco municípios selecionados, e discutir as relações observadas entre os componentes de governo, as características da gestão e as práticas de saúde.

\section{Metodologia}

\section{Seleção dos casos}

Foram realizados cinco estudos de caso com níveis de análise imbricados 21 , em municípios do Estado da Bahia, todos em gestão plena do sistema de saúde. Os critérios usados para seleção dos municípios foram os seguintes: (a) estar habilitado na gestão plena do sistema pela NOB96; (b) apresentar continuidade político-administrativa nos últimos seis anos; (c) ter algumas características sócio-demográficas e de saúde equivalentes, tais como população, urbanização e cobertura do Programa Saúde da Família (Tabela 1). Além disso, dos cinco municípios, dois foram escolhidos por serem considerados "exitosos" e dois "não-exitosos" em relação ao cumprimento das diretrizes do Sistema Único de Saúde (SUS) na descentralização, de acordo com a percepção de diversos informantes entrevistados, pertencentes à Secretaria Estadual de Saúde e ao Conselho Nacional de Secretários Municipais de Saúde (CONASEMS) 22. Quatro municípios preencheram todos os critérios inclusive o piloto. O Município B, apesar de possuir menor porte populacional foi incluído por estar na gestão plena, com continuidade administrativa e ser considerado como experiência exitosa pelos informantes-chave.

A necessidade de se analisar diferentes níveis da realidade decorreu da complexidade do objeto - o município. Esses níveis correspondem a partes do caso a ser investigado que estão relacionadas, "imbricadas", mas que podem ser estudadas de forma separada e que se complementam.

\section{Modelo lógico}

Para a seleção das dimensões e critérios para a avaliação foi elaborado um modelo teórico-lógico 23,24, correspondente a uma "imagem-objetivo” da reorganização das práticas assistenciais, na direção das diretrizes constitucionais do SUS, relacionada ao processo de descentralização da saúde em municípios. Essa "imagem-objetivo" funcionou como referência para apreciação do grau de implantação dos componentes da descentralização avaliados (Figura 1).

A descentralização na gestão da saúde foi considerada, no presente estudo, como a transferência de poder administrativo e técnico do âmbito federal para o âmbito municipal formalizada por meio da habilitação na gestão plena do sistema pela NOB-96. Num Estado federativo como o Brasil, esse processo deve considerar os três âmbitos do poder: o espaço geral, referente à situação nacional, o espaço particular relaciona- 
Pontuação de níveis, dimensões e critérios para a análise de implantação da gestão descentralizada do Sistema Único de Saúde no Brasil, 2002.

\begin{tabular}{|c|c|c|c|}
\hline \multicolumn{4}{|c|}{ Níveis, dimensões e critérios (pontos máximos) } \\
\hline $\begin{array}{l}\text { I. Componentes do } \\
\text { governo (100 pts.) * }\end{array}$ & II. Gestão da saúde (100 pts.) * & \multicolumn{2}{|c|}{ III. Práticas assistenciais (200 pts.) * } \\
\hline 1. Projeto de governo (35 pts.) ** & 1. Planejamento e avaliação & 1. Organização das práticas & 1.1.5. Atenção à saúde bucal (6 pts.) \# \\
\hline 1.1. Plano de governo (11 pts.) $* \star \star$ & $(20 \text { pts. })^{\star \star}$ & $(100$ pts. $) * \star$ & a) Programação e intervenção sobre \\
\hline & 1.1. Características do planejamento & 1.1. Integralidade da atenção à saúde & \\
\hline prioridade no plano de governo e no & $(10 \text { pts. })^{\star \star \star}$ & $(55 \text { pts.) })^{\star \star \star}$ & a saúde bucal a partir do perfil \\
\hline discurso dos entrevistados (6 pts.); & a) Práticas de planejamento & 1.1.1. Ações de promoção da saúde & epidemiológico (3 pts.); \\
\hline b) No plano de saúde há coerência & situacional local em saúde (5 pts.); & $(13$ pts.) \# & b) Ênfase nas ações preventivas e \\
\hline entre problemas priorizados e ações & b) O planejamento é regular e usado & a) Existência de ações intersetoriais & coletivas (2 pts.); \\
\hline propostas (5 pts.). & como instrumento de gestão (4 pts.); & voltadas para os problemas & c) Disponibilidade de atendimento \\
\hline 1.2. Financiamento para a saúde & c) O planejamento é normativo (1 pt.). & priorizados no território (7 pts.); & restaurador (1 pt.). \\
\hline$(12 \text { pts.) })^{\star \star \star}$ & 1.2. Institucionalização da avaliação & b) Incorporação de tecnologias de & 1.2. Cuidado integral (45 pts.) \# \\
\hline a) Gastos com saúde: percentual do & $(10 \text { pts. })^{\star \star \star}$ & comunicação social (6 pts.). & 1.2.1. Protocolos de atenção para \\
\hline orçamento executado do tesouro & a) Existem responsáveis que realizam & 1.1.2. Ações de vigilância de riscos e & agravos específicos priorizados \\
\hline municipal previsto na Emenda & regularmente a avaliação e o produto & danos à saúde (12 pts.) \# & $(15$ pts.) \# \\
\hline Constitucional 29 (6 pts.) & é usado na tomada de decisões & a) Utilização dos dados dos sistemas & a) Elaboração de protocolos \\
\hline (Se o gasto é acima de 8\%: 6 pts.; & $(5$ pts.); & de informação para monitoramento & específicos de nível local (8 pts.); \\
\hline entre 7 e $8 \%$ : 4 pts.; abaixo de $7 \%$ : & b) Os gestores e técnicos utilizam & da situação de saúde e tomada de & b) Utilização sistemática dos \\
\hline 2 pts.); & dados dos sistemas de informação & decisões no nível local (6 pts.); & protocolos de atenção para agravos \\
\hline b) Gastos per capita em R\$ e US\$ & oficiais na monitorização e outras & b) Estruturação de um sistema de & de prioridade estadual/nacional \\
\hline (6 pts) & avaliações pontuais (5 pts.). & vigilância da saúde com integração/ & \\
\hline (Se $\geq$ US\$150 per capita: 6 pts.; & 2. Organização do sistema (56 pts.) ** & articulação entre as ações de & 1.2.2. Estratégias de acolhimento \\
\hline$<$ US\$150 e $\geq$ US\$80: 4 pts.; & 2.1. Relação público-privado & vigilância epidemiológica e vigilância & $(15$ pts.) \# \\
\hline$<$ US\$80: 2 pts.). & $(10$ pts. $) \star \star \star ~$ & & a) Instalações física \\
\hline 1.3. Iniciativas intersetoriais & a) Ações de média e alta & 1.1.3. Articulação dos níveis de & mínimo (bancos e cadeiras suficientes, \\
\hline$(12 \text { pts.) })^{\star \star \star}$ & complexidade geridas pelo setor & prevenção, recuperação e reabilitação & área coberta com acesso a banheiros \\
\hline a) Planejamento integrado/comitê & público (4 pts.); & da saúde e de controle dos & e água potável e gar \\
\hline gestor misto (4 pts.); & b) Existência de serviços públicos de & problemas de saúde locais (12 pts.) \# & privacidade do atendimento) (7 pts.); \\
\hline b) Orçamento próprio para os & referência para regulação da oferta & a) Existência de estratégias do & b) Utilização de diálogo e fle \\
\hline projetos (4 pts.); & $(3$ pts.); & diagnóstico precoce e prevenção, & na rotina da rede de serviços (4 pts.); \\
\hline c) Existência de, pelo menos, um & c) Ações de média e alta & pelo menos para grupos de risco da & c) Triagem realizada por profissional \\
\hline projeto implantado (4 pts.). & complexidade referenciadas para & hipertensão arterial (4 pts.); & \\
\hline 2. Capacidade de governo (30 pts.) ** & o setor privado contratado e & b) Integração dos níveis de & 1.2.3. Práticas com equipes \\
\hline 2.1. Perfil da direção (10 pts.) ${ }^{\star \star \star}$ & conveniado (3 pts.). & prevenção, recuperação e reabilitação & multidisciplinares (15 pts.) \# \\
\hline a) Existência de liderança ou quadro & 2.2. Adequação da oferta às & para os agravos/problemas & a) Atividades de grupo (terapêuticos, \\
\hline político na direção do setor saúde & necessidades da população & priorizados em cada município & preventivos, e educativos) (5 pts.); \\
\hline$(4$ pts.); & $(10 \text { pts. })^{\star \star \star}$ & $(4$ pts.); & b) Projetos terapêuticos integrados e \\
\hline b) Formação na área de saúde & a) Incorporação de ações & c) Coerência entre as ações de & centrados no usuário (5 pts.); \\
\hline coletiva do dirigente (3 pts.); & programáticas de saúde e oferta & prevenção e controle (ações sobre & c) Utilização de protocolos nas \\
\hline c) Concepção de um sistema de & de serviços adequada ao perfil & os riscos) realizadas e os problemas & atividades realizadas pela equipe \\
\hline saúde local universal e de qualidade & epidemiológico e social (6 pts.); & priorizados (4 pts.). & (5 pts.) \\
\hline (3 pts.). & b) Incorporação de ações & 1.1.4. Assistência farmacêutica & 2. Efetividade das práticas \\
\hline 2.2. Adequação do quadro técnico às & programáticas exclusivamente dos & $(12$ pts.) \# & $(100$ pts. $) * \star$ \\
\hline necessidades do sistema municipal de & programas definidos pelo nível & a) Disponibilidade de medicamentos & 2.1. Efeitos relacionados à saúde \\
\hline saúde (10 pts.) $)^{\star \star \star}$ & federal e oferta de serviços sem & para doenças de maior prevalência & $(70 \text { pts. })^{\star \star \star}$ \\
\hline a) Quadros técnicos qualificados por & relação com o perfil epidemiológico & e/ou gravidade/custo (6 pts.); & \\
\hline instituições de saúde coletiva (4 pts.); & (atendimento prioritário da demanda & b) Desenvolvimento de ações & \\
\hline b) Número adequado de profissionais & espontânea) (4 pts.). & de promoção do uso racional de & \\
\hline por área de atuação (3 pts.); & & & \\
\hline
\end{tabular}

(continua) 


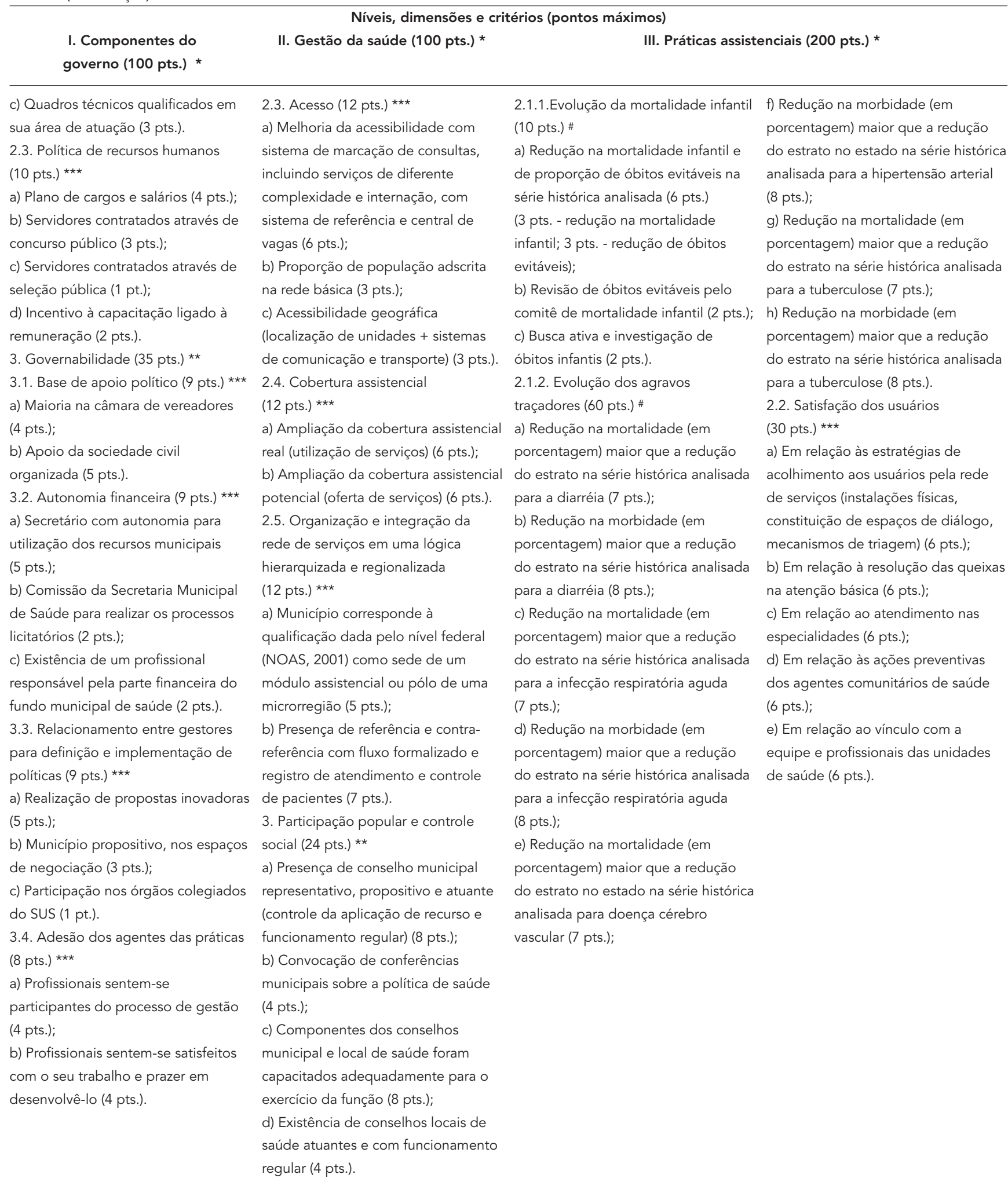

Pts. = pontos.

* Total de pontos $=\Sigma$ Subtotais;

** Subtotal $=\Sigma$ Subtotais 1 ;

$\star \star \star$ Subtotal $1=\Sigma$ Subtotais 2 ;

\# Subtotais 2. 


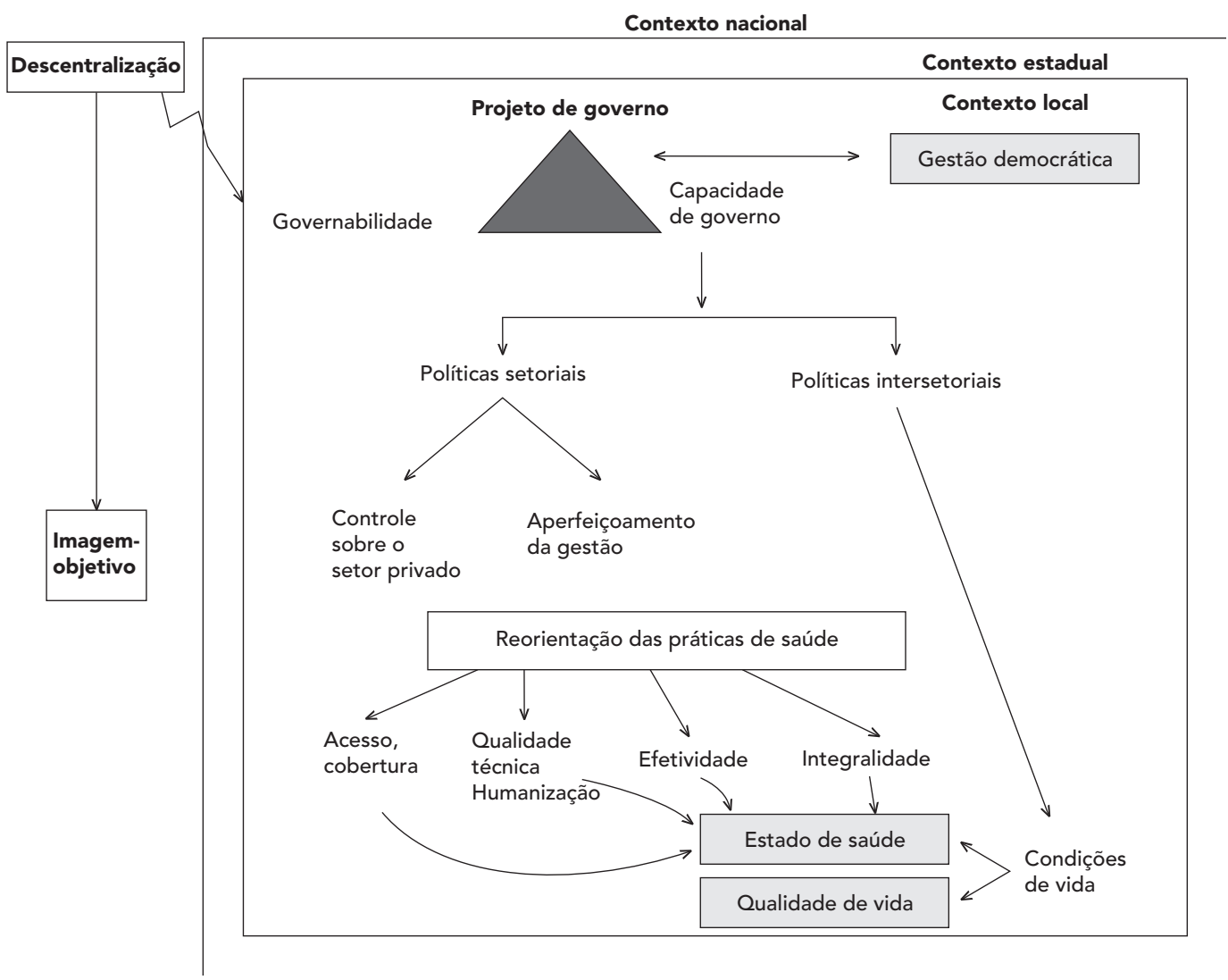

do à situação estadual e o espaço singular 25 do município em estudo (Figura 1).

No espaço singular do município, onde a descentralização se efetiva, três componentes foram examinados: (a) projeto de governo; (b) capacidade de governo e (c) governabilidade. O projeto de governo se refere ao conjunto de proposições elaboradas pelos dirigentes locais visando ao alcance dos seus objetivos. A capacidade de governo corresponderia aos recursos relacionados com a condução e gerenciamento adequado das ações. Já a governabilidade diz respeito às condições necessárias a implementação das ações propostas, considerando os obstáculos e oportunidades que o meio oferece a essas propostas 25 .

De acordo com a "imagem-objetivo" concebida, poder-se-ia admitir que a descentralização, quando ocorre em adequada articulação entre os componentes do triângulo de governo, corresponderia a um fortalecimento do poder local, das instituições públicas e da capacidade de gestão dos sistemas de saúde. A atuação conjunta dos três componentes pode resultar em melhor organização dos serviços de saúde viabilizando maior acesso e cobertura; também as condições seriam favoráveis a um maior controle sobre os prestadores privados, com otimização do uso dos recursos públicos visando a assegurar maior integralidade e efetividade. Essa situação é favorável a uma maior participação popular, o que poderia aproximar os gestores das necessidades da população e melhorar a capacidade de intervenção do sistema.

Com uma melhor gestão da saúde, o propósito de reorientação das práticas de assistência individual e coletiva poderia ser potencializado. Destaca-se naquele nível, a articulação de iniciativas relacionadas com a humanização do acolhimento e aquelas relativas ao aumento da efetividade dos problemas de saúde por meio de 
uma melhoria na qualidade técnico-científica do cuidado. Por outro lado, a inclusão de projetos intersetoriais, no âmbito da formulação de políticas públicas saudáveis, envolvendo além da saúde a esfera do trabalho, da educação, da comunicação e da infra-estrutura urbana, poderia viabilizar ações de alcance coletivo voltadas para a promoção da saúde. Esse conjunto de intervenções poderia contribuir para uma melhoria no estado de saúde da população por meio da redução dos indicadores de internação hospitalar e dos coeficientes de mortalidade, além de gerar maior satisfação dos usuários com os serviços de saúde, garantindo, portanto, melhor qualidade de vida para os mesmos (Figura 1).

\section{Níveis, dimensões e critérios}

Foram selecionados para análise os seguintes níveis: (i) componentes do governo; (ii) gestão da saúde e (iii) práticas assistenciais. Para cada nível, foram selecionadas dimensões, subdimensões e critérios a partir de "imagem-objetivo", que foram sumarizados numa matriz contendo a descrição do padrão esperado para cada um dos critérios (Tabela 1). Essa matriz foi submetida a um grupo de dirigentes e técnicos do Ministério da Saúde para uma atribuição inicial de pesos, utilizando-se a técnica do grupo nominal 26.

Para analisar o nível componentes do governo, foram escolhidos critérios para cada uma das dimensões propostas. Para o projeto de governo foram selecionados os seguintes critérios: (a) prioridade para saúde - foi verificado se a mesma estava explicitada tanto no plano de governo quanto no discurso dos entrevistados. Além disso, observou-se no plano municipal de saúde se havia consistência entre problemas priorizados e ações propostas; (b) características do financiamento setorial - foi obtido o percentual de gastos com saúde em relação ao total de receitas de tributos do município. Foram classificados como: incipientes - os municípios cujo percentual de gastos com saúde foi inferior a 7\%; intermediários - os municípios cujo percentual de gastos com saúde situava-se entre $7 \%$ e $8 \%$; e avançados - os municípios cujo percentual de gastos com saúde foi superior a $8 \%$. Foi ainda calculado o gasto público per capita em saúde, em Reais (R\$) e em Dólar (US\$). Foi feita a conversão de real para dólar considerando uma relação de 2:1. Foi calculada a razão entre o gasto total em saúde (gasto municipal, estadual e federal) e a população total do município para aquele ano. Foram classificados como: incipientes - os municípios com gasto per capita inferior a US\$80; intermediários - os municípios com gasto per capita entre US\$80 e US\$150; avançados - municípios com gasto per capita superior a U\$150. Terceiro critério: (c) existência de iniciativas intersetoriais - foram consideradas iniciativas intersetoriais aquelas ações articuladas entre a saúde e outros setores, para as quais existisse ou planejamento integrado ou comitê gestor misto entre dois ou mais setores do governo, com orçamento próprio para os projetos. Verificou-se se havia, pelo menos, um projeto implantado.

Em relação à capacidade de governo foram escolhidos os seguintes os critérios: (a) perfil do gestor - analisado no que diz respeito à liderança e à formação na área de saúde coletiva; (b) adequação do quadro técnico às necessidades do Sistema Municipal de Saúde - verificou-se se os assessores diretos possuíam formação ou especialização em saúde coletiva, se havia um número adequado de profissionais qualificados em suas áreas específicas de atuação; (c) política de recursos humanos - existência de um plano de cargos e salários, contratação por meio de concurso público ou seleção pública, incentivo à capacitação ligado à remuneração ou, pelo menos, iniciativas de capacitação sem vínculo com remuneração.

Quanto à governabilidade foram os seguintes os critérios: (a) existência de base de apoio políti$\mathrm{co}$ - base de apoio parlamentar e da sociedade civil organizada; (b) autonomia financeira do setor saúde em relação à prefeitura - existência de comissão própria da Secretaria Municipal de Saúde para realizar os processos licitatórios, bem como de profissional responsável pela gestão financeira do Fundo Municipal de Saúde; (c) características do relacionamento entre gestores - articulação entre os níveis de gestão e busca de inovação na gestão; (d) adesão dos profissionais à gestão - satisfação dos profissionais com o trabalho desenvolvido (realização profissional e pessoal) e percepção de "participação" na gestão.

Para analisar o nível gestão da saúde, na dimensão planejamento, verificou-se: (a) características do planejamento - práticas de planejamento situacional local em saúde, regularidade do planejamento e sua utilização como instrumento de gestão; (b) institucionalização da avaliação - existência ou não de avaliação e suas características (se realizada de forma regular e utilizada para a tomada de decisões; se os sistemas de informação oficiais eram usados no monitoramento das ações implementadas).

Em relação à dimensão organização do sistema foram investigados: (a) relação público-priva$d o$ - foi verificado se a gestão das ações de média e alta complexidade era feita pelo setor público, se existiam serviços públicos de referência para regulação da oferta; (b) adequação da oferta às necessidades da população - análise sobre a incorporação de ações programáticas de saúde e 
oferta de serviços, se a mesma era adequada ao perfil epidemiológico e social ou, pelo menos, se havia implementação dos programas definidos pelo nível federal; (c) acesso - existência de sistema de marcação de consultas, incluindo serviços de diferente complexidade e internação, com sistema de referência e central de vagas; aumento da proporção de população adscrita na rede básica e melhoria da acessibilidade geográfica, ou seja, distribuição espacial das unidades ou existência de sistemas de comunicação e transporte; (d) cobertura assistencial - a cobertura assistencial potencial (oferta de serviços) foi estimada por meio do número de unidades de saúde, bem como da concentração de profissionais/população (médicos, enfermeiros, odontólogos e pessoal auxiliar). Já a cobertura assistencial real (utilização de serviços) foi estimada utilizando-se a produção ambulatorial do SUS, por ano de competência, pela população, no período compreendido entre 1994 e 2001; (e) organização e integração da rede de serviços em uma lógica hierarquizada e regionalizada - adequação entre a qualificação dada pelo nível federal de acordo com a Norma Operacional de Atenção à Saúde (NOAS) 13, como sede de um módulo assistencial ou pólo de uma microrregião, e a existência de um sistema de referência e contrareferência com fluxo formalizado, registro de atendimento e controle de pacientes; (f) participação popular e controle social - participação da sociedade nas decisões sobre a política de saúde, buscando-se observar a presença de conselhos municipal e local representativos, propositivos e atuantes (controle da aplicação de recurso e funcionamento regular); a convocação de conferências municipais sobre a política de saúde; a existência de capacitação para os membros dos conselhos municipal e local de saúde.

Finalmente, em relação ao nível das práticas assistenciais, foram analisadas duas dimensões: organização e resultados. No que diz respeito à organização das práticas, duas subdimensões da integralidade da atenção à saúde foram consideradas. A primeira relacionada com a oferta, articulação e continuidade da atenção nos diversos níveis de complexidade do sistema necessários ao controle de danos, riscos e causas. Nessa direção, verificou-se a existência de: (a) ações de promoção da saúde - ações intersetoriais voltadas para os problemas priorizados no território, incorporação por parte do nível central e local de tecnologias de comunicação social; (b) ações de vigilância de riscos e danos à saúde - utilização dos dados dos sistemas de informação para monitoramento da situação de saúde e tomada de decisões no nível local, e sobre a integração/articulação entre as ações de vigilância epidemioló- gica e vigilância sanitária; (c) articulação entre as ações de promoção, proteção, recuperação e reabilitação da saúde - integração entre essas ações para os agravos/problemas priorizados no município. Existência de estratégias de diagnóstico precoce e prevenção pelo menos para grupos de risco de hipertensão arterial, coerência entre as ações de prevenção e controle (ações sobre os riscos) realizadas e os problemas priorizados.

Já a dimensão cuidado integral, refere-se à articulação entre as ações de promoção, proteção e recuperação no nível do indivíduo. Os critérios para avaliação desse componente foram: (a) estratégias de acolhimento - iniciativas referentes à melhoria do acolhimento aos usuários. Incluem instalações físicas com conforto mínimo (bancos e cadeiras suficientes, área coberta com acesso a banheiros, água potável e garantia à privacidade no atendimento), existência de diálogo e flexibilidade na rotina da rede de serviços e triagem realizada por profissional de saúde; (b) práticas com equipes multiprofissionais - articulação entre o trabalho dos diversos componentes da equipe de saúde. Existência de trabalho com grupos de usuários (terapêuticos e educativos) e com equipes multiprofissionais; (c) existência de protocolos de atenção para agravos específicos priorizados, protocolos específicos para o nível local ou, pelo menos, a utilização sistemática dos protocolos de atenção para agravos de prioridade estadual/nacional; (d) assistência farmacêutica - disponibilidade de medicamentos para doenças de maior prevalência e/ou gravidade/custo e desenvolvimento de ações de promoção do uso racional de medicamentos; (e) atenção à saúde bucal - verificou-se a existência de programação e intervenção sobre os problemas relacionados com a saúde bucal a partir do perfil epidemiológico do município. Ênfase nas ações preventivas e coletivas. Disponibilidade de atendimento restaurador.

\section{Avaliação da efetividade das práticas}

A efetividade das práticas foi aqui considerada como o efeito de uma intervenção em um sistema operacional 27. Para a sua avaliação, foram analisadas as séries temporais de agravos, considerados como condições traçadoras, selecionadas conforme critérios propostos por Kessner et al. 28, no período compreendido entre 1980 e 1999 para os municípios estudados. Foram os seguintes os indicadores selecionados: (a) coeficiente de mortalidade infantil; (b) coeficiente de mortalidade infantil evitável; (c) proporção de óbitos infantis evitáveis; (d) mortalidade infantil por diarréia; (e) mortalidade infantil por infecção respiratória aguda; (f) coeficiente padronizado 
de mortalidade por tuberculose e coeficiente padronizado de mortalidade por doença cérebro vascular. Em seguida foi analisada a evolução das médias qüinqüenais dos coeficientes de mortalidade dos agravos considerados como traçadores, entre 1990 e 1999. Como valor de referência foram consideradas as médias qüinqüenais dos coeficientes de mortalidade para os mesmos agravos referentes ao estrato do qual o município-caso fazia parte.

Os seguintes indicadores de morbidade para os municípios-caso no período compreendido entre 1995 e 2001 foram escolhidos: (a) taxa de internação hospitalar por causa específica (tuberculose e hipertensão arterial); (b) taxa de internação hospitalar por diarréia e infecção respiratória aguda, para menores de um ano. Esses indicadores foram comparados com a mesma taxa do estrato de municípios de referência.

A análise foi feita por meio da inspeção visual das curvas da série histórica frente a fatos e eventos relacionados com o processo de descentralização no município. Para melhor visualização da tendência, as variabilidades verificadas nessas curvas foram suavizadas pelo emprego do método das médias móveis de ordem 5 no caso da mortalidade, e de ordem 3 para os internamentos. Calculou-se também a velocidade da variação média anual $(\beta)$ dos coeficientes para todo o período analisado, utilizando-se a análise de regressão linear bivariada, na qual o ano calendário foi a variável independente. Além disso, estimou-se a variação percentual $(\Delta \%)$ anual e entre as médias qüinqüenais dos referidos indicadores. Foi considerada como avançada a evolução, cujo desempenho desses indicadores tivesse sido melhor que aquele calculado para o estrato de municípios com situação sócio-econômica semelhante (Tabela 1).

\section{Fontes e coleta de dados}

A coleta de dados foi realizada por meio de: (1) análise documental dos planos de saúde 19982001, relatórios de gestão 1999 e 2000 e repasses federais aos municípios entre 1997 e 2001; (2) os dados de morbidade foram obtidos no Sistema de Informação Hospitalar do Sistema Único de Saúde (SIH-SUS) no período de 1995 a 2001; (3) os dados de mortalidade foram obtidos do Sistema de Informação de Mortalidade do Sistema Único de Saúde (SIM-SUS) no período de 1980 a 1999; (4) entrevistas semi-estruturadas a partir de um roteiro de entrevistas abrangendo as dimensões e critérios selecionados; (5) diários de campo, onde se encontram o registro das observações e impressões das entrevistas, estratégias de pesquisa e outras questões consideradas relevantes; e (6) a produção ambulatorial para estimativa da cobertura assistencial foi obtida do Sistema de Informação da Atenção Básica (SIAB) e SIH-SUS

Duas unidades de saúde caracterizadas como "satisfatória" e "não-satisfatória", identificadas com base em entrevistas abertas com técnicos do nível central das Secretarias Municipais de Saúde nos cinco municípios, foram selecionadas para observação e realização das entrevistas. Foram realizadas 101 entrevistas com os seguintes informantes: gestor do sistema (seis); técnicos do nível central (13); profissionais de saúde das unidades (29); agentes comunitários de saúde (11) e cerca de quatro usuários do serviço da unidade de observação considerada "satisfatória" e também das unidades de observação consideradas "não-satisfatórias", num total de 42 usuários entrevistados nos cinco municípios, sendo que destes, um era representante dos usuários ou prestadores de serviço no Conselho Municipal de Saúde.

Foi obtido consentimento informado verbal dos entrevistados e o sigilo, tanto dos entrevistados quanto dos municípios, foi assegurado.

\section{$\underline{\text { Análise }}$}

A matriz contendo dimensões e critérios para a avaliação foi submetida a um comitê de experts, para validação através da realização de uma conferência de consenso que recorreu a uma técnica mista (Delphi e presencial), segundo a metodologia descrita por Souza et al. ${ }^{29}$. As entrevistas foram transcritas e analisadas segundo as dimensões propostas. O grau de aproximação entre a situação observada e a "imagem-objetivo" foi denominado "grau de implantação". O mesmo foi estimado para cada critério, dimensão e nível de análise por meio de um sistema de ponderação baseado tanto na revisão da literatura sobre modelos assistenciais quanto subsidiado pelos resultados da conferência de consenso. Foram atribuídos 100 pontos para os níveis de análise "componentes do governo" e "gestão da saúde", e 200 pontos para aquele das "práticas assistenciais", totalizando 400 pontos. Esses pontos foram distribuídos pelas dimensões, subdimensões e critérios selecionados de acordo com sua importância relativa (Tabela 1). A atribuição de pontos a partir do material empírico colhido foi feita por dois pesquisadores isoladamente, a partir da leitura das entrevistas, da análise documental e de dados secundários. Os casos onde houve discordância foram revistos visando à obtenção do consenso entre os pesquisadores. A classificação do grau de implantação, para cada critério, foi feita a partir da pontuação atribuída, e correspondeu à diferença percentual entre a 
pontuação máxima e aquela atribuída pelos investigadores de acordo com os seguintes pontos de corte: (1) incipiente: $\geq 0$ e $\leq 33,3 \%$; (2) intermediário: > 33,3 e $\leq 66,6 \%$; (3) avançado: > 66,6\% 30 .

Baseando-se nos resultados obtidos foi feita uma comparação entre os cinco municípios estudados, visando a identificar a concordância entre os escores registrados nos três níveis de análise. Foi então indagado: (a) em que medida havia concordância entre as características de governo e da gestão?; (b) em que medida o grau de organização do setor saúde concordava com o grau de organização das práticas?; (c) por fim, foi analisado se havia coerência entre a organização das práticas de saúde e os efeitos observados (Tabela 2).

\section{Resultados}

\section{Algumas características sócio-demográficas e de saúde dos municípios-caso}

Todos os municípios estudados apresentavam um percentual de população com renda insuficiente superior a 50\% e, à exceção do Município B, possuíam uma população superior a 100 mil habitantes, com percentuais de urbanização muito próximos, apesar das diferenças em termos de densidade demográfica. $\mathrm{O}$ gasto público total com saúde per capita, foi muito semelhante no ano 2000 entre os Municípios C, D e E, mais elevado no Município A e mais baixo no Município B. Observa-se também semelhança nos per-

Tabela 2

Grau de implantação (n e \%) segundo níveis, dimensões e subdimensões da gestão descentralizada do Sistema Único de Saúde (SUS) em cinco municípios da Bahia, Brasil, 2001.

\begin{tabular}{|c|c|c|c|c|c|c|c|c|c|c|c|c|}
\hline \multirow{3}{*}{$\begin{array}{l}\text { Níveis, } \\
\text { dimensões } \\
\text { e subdimensões }\end{array}$} & \multicolumn{10}{|c|}{ Municípios } & \multirow{2}{*}{\multicolumn{2}{|c|}{$\begin{array}{l}\text { Pontuação } \\
\text { máxima }\end{array}$}} \\
\hline & \multicolumn{2}{|c|}{ A } & \multicolumn{2}{|c|}{ B } & \multicolumn{2}{|c|}{ C } & \multicolumn{2}{|c|}{ D } & \multicolumn{2}{|c|}{$\mathbf{E}$} & & \\
\hline & $\mathrm{n}$ & $\%$ & n & $\%$ & $\mathrm{n}$ & $\%$ & $\mathbf{n}$ & $\%$ & n & $\%$ & $\mathrm{n}$ & $\%$ \\
\hline \multicolumn{13}{|l|}{ I. Componentes de governo } \\
\hline 1. Projeto de governo & 16 & $45,7^{\star \star}$ & 14 & 40,0 ** & 21 & $60,0 * *$ & 13 & $37,1 * *$ & 13 & $37,1 * \star$ & 35 & 100,0 \\
\hline 2. Capacidade de governo & 10 & 33,3 * & 10 & 33,3 * & 18 & 60,0 ** & 6 & 20,0 * & 8 & 26,7 * & 30 & 100,0 \\
\hline 3. Governabilidade & 23 & $65,7^{\star \star}$ & 33 & $94,3 * \star \star$ & 35 & $100,0 * \star \star$ & 11 & 31,4 * & 18 & 51,4 ** & 35 & 100,0 \\
\hline Subtotal a & 49 & $49,0 * \star$ & 57 & $57,0 * \star$ & 74 & $74,0 \star \star \star$ & 30 & 30,0 * & 39 & $39,0 * *$ & 100 & 100,0 \\
\hline \multicolumn{13}{|l|}{ II. Gestão da saúde } \\
\hline 1. Planejamento e avaliação & 2 & 10,0 * & 2 & 10,0 * & 6 & 30,0 * & 0 & 0,0 * & 1 & 5,0 * & 20 & 100,0 \\
\hline 2. Organização do sistema & 28 & $50,0 \star \star$ & 32 & $57,1 \star \star$ & 53 & $94,6 * \star \star$ & 15 & 26,8 * & 26 & 46,4 ** & 56 & 100,0 \\
\hline 3. Participação e controle social & 12 & $50,0 * \star$ & 6 & 25,0 * & 22 & $91,7 \star \star \star$ & 8 & 33,3 * & 12 & $50,0 * \star$ & 24 & 100,0 \\
\hline Subtotal b & 42 & $42,0 * \star$ & 40 & $40,0 * \star$ & 81 & $81,0 * \star \star$ & 23 & 23,0 * & 39 & $39,0 * \star$ & 100 & 100,0 \\
\hline \multicolumn{13}{|l|}{ III. Práticas assistenciais } \\
\hline \multirow{2}{*}{\multicolumn{13}{|c|}{$\begin{array}{l}\text { 1. Organização das práticas } \\
\text { assistenciais }\end{array}$}} \\
\hline & & & & & & & & & & & & \\
\hline \multicolumn{13}{|l|}{ 1.1. Integralidade } \\
\hline da atenção & 19 & 34,5 ** & 7 & 12,7 * & 35 & $63,6 * *$ & 10 & 18,2 * & 14 & 25,5 * & 55 & 100,0 \\
\hline 1.2. Cuidado integral & 8 & 17,8 * & 8 & 17,8 * & 30 & 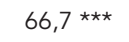 & 8 & 17,8 * & 6 & 13,3 * & 45 & 100,0 \\
\hline Subtotal c & 27 & 27,0 * & 15 & 15,0 * & 65 & $65,0 * *$ & 18 & 18,0 * & 20 & 20,0 * & 100 & 100,0 \\
\hline \multicolumn{13}{|l|}{ 2. Resultados das } \\
\hline \multicolumn{13}{|l|}{ práticas assistenciais } \\
\hline 2.2. Efetividade & 47 & 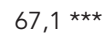 & 15 & 21,4 * & 55 & 78,6 *** & 32 & $45,7^{\star \star}$ & 23 & 32,9 * & 70 & 100,0 \\
\hline \multicolumn{13}{|l|}{ 2.3. Satisfação } \\
\hline dos usuários & 12 & 40,0 ** & 14 & $46,7^{\star \star}$ & 24 & $80,0 \star \star \star$ & 10 & 33,3 * & 10 & 33,3 * & 30 & 100,0 \\
\hline Subtotal d & 59 & $59,0 * \star$ & 29 & 29,0 * & 79 & $79,0 \star \star \star$ & 42 & $42,0 * \star$ & 33 & 33,0 * & 100 & 100,0 \\
\hline Subtotal $c+d$ & 86 & 43,0 ** & 44 & 22,0 * & 144 & $72,0 * \star \star$ & 60 & 30,0 * & 53 & 26,5 * & 200 & 100,0 \\
\hline Escore global $(a+b+c+d)$ & 177 & 44,3 ** & 135 & 33,8 ** & 299 & $74,8 * \star \star$ & 113 & 28,3 * & 131 & 32,8 * & 400 & 100,0 \\
\hline
\end{tabular}

* Incipiente;

** Intermediário;

*** Avançado. 
centuais de cobertura do Programa de Agentes Comunitários de Saúde (PACS) entre aqueles de porte populacional semelhante como os Municípios A, C e D. O coeficiente de mortalidade infantil apresentou diferenças entre os municípios, variando entre $18 /$ mil nascidos vivos no Município D e $42 /$ mil nascidos vivos no Município E. Nesse último caso, bastante superior à média nacional de 33/mil nascidos vivos. A proporção dos óbitos infantis por causas mal definidas foi bastante alta nos Municípios B, D e E e baixa nos Municípios C (11,7\%) e A (3\%) em 1999 (Tabela 3).

O grau de implantação da gestão descentralizada do SUS

A comparação entre os cinco casos revela, em geral, uma concordância entre o estágio de implantação da descentralização, as características de governo, da gestão e os resultados obtidos sobre as práticas assistenciais. Assim, a uma classificação "avançada” para a descentralização (escore global do Município C com $74,8 \%$ ), corresponderam componentes do governo (74\%), gestão do setor saúde (81\%) e práticas assistenciais "avançadas" (72\%). No outro extremo do espectro, a uma classificação global incipiente (Município D, com 28,3\%) corresponderam componentes do governo (30\%), gestão da saúde (23\%) e práticas assistenciais também incipientes (30\%). Da mesma forma, num dos municípios classificados como em estágio intermediário (Município A), a classificação também foi intermediária para os três níveis de análise: componentes do governo (49\%), gestão da saúde (2\%) e práticas assistenciais (43\%). Já nos outros dois municípios a concordância foi parcial (Tabela 2, Figura 2).

Houve maior grau de concordância entre os escores atribuídos aos componentes de governo e aqueles atribuídos à gestão da saúde, nos cinco municípios, ou seja, nos dois municípios intermediários (A e B), a um "governo" considerado como intermediário correspondeu uma "gestão" também intermediária. Da mesma forma, no Município C, os dois níveis de análise foram classificados como avançados e no Município D os dois níveis foram incipientes. A comparação entre as características da gestão da saúde e as práticas assistenciais revelou um resultado consistente nos Municípios A, C e D. Já nos Municípios B e E, a uma gestão intermediária corresponderam práticas incipientes (Tabela 2). Quando se analisa a relação entre o escore global dos municípios e o efeito das ações sobre as práticas, verifica-se concordância nos Municípios C e E, resultados inversos em A e D e resultado paradoxal em B, onde os efeitos foram classificados como inci- pientes e o município estava num estágio intermediário.

O Município C foi o único classificado como "avançado" em relação à "imagem-objetivo" delimitada, em todos os níveis analisados (Tabela 2, Figura 2). Nesse município, existia um claro projeto de reorganização da rede municipal, desde 1997, conduzido durante duas gestões sucessivas pelo secretário de saúde e prefeito. Concomitantemente, a capacidade de governo do secretário municipal de saúde, tanto técnica como política, resultava não apenas de sua titulação formal (mestrado em Saúde Coletiva) como da experiência profissional anterior. Aliado a isso, somava-se o fato de o gestor constituir-se em liderança política com militância no movimento da reforma sanitária, liderança esta amplamente reconhecida pelos diversos entrevistados. A governabilidade era dada tanto pelo apoio político formal que o gestor municipal possuía quanto pela autonomia da Secretaria Municipal em relação à utilização dos recursos financeiros do SUS. Esse quadro, possivelmente propiciou a reorganização da rede assistencial com maior controle sobre o setor privado, com redução das internações desnecessárias e ênfase na atenção básica com ampliação da cobertura, a partir de ações programáticas e melhoria da acessibilidade com a implantação de central de marcação de consultas.

Em contrapartida, no extremo oposto, os $\mathrm{Mu}$ nicípios D e E, classificados como incipientes, não apresentavam claro projeto de reorganização do setor saúde, seja nos planos de saúde, seja no discurso de seus gestores e técnicos. No Município D a descentralização era vista pelo gestor como uma possibilidade de "desresponsabilização" por parte do governo federal. Embora houvesse razoável governabilidade em $\mathrm{E}$, ambos os municípios não dispunham de quadros técnicos com formação específica e ou experiência para a gestão de um Sistema Municipal de Saúde.

A despeito dessas diferenças, verifica-se que tanto o projeto de governo quanto a capacidade de governo não atingiram o estágio avançado em nenhum dos municípios estudados (Tabela 2). A análise em profundidade revelou problemas relacionados com ausência ou limitação nas iniciativas intersetoriais e inexistência de política de recursos humanos claramente definida (entrevistas com gestores).

Chama a atenção aspectos que foram incipientes em todos os municípios como o planejamento e a avaliação. O planejamento nos Municípios D e E era fundamentalmente normativo e feito de forma assistemática. Os planos se constituíam em documentos cartoriais e não eram utilizados como guia para a ação. Já nos Municí- 
Características sócio-demográficas, sanitárias e de saúde em cinco municípios da Bahia, Brasil, 2001.

\begin{tabular}{|c|c|c|c|c|c|}
\hline \multirow[b]{2}{*}{ Indicadores } & \multicolumn{5}{|c|}{ Municípios } \\
\hline & A & B & C & D & $\mathrm{E}$ \\
\hline População aproximada * & 165.000 & 20.000 & 260.000 & 140.000 & 100.000 \\
\hline Porcentagem de urbanização (habitação em zona urbana) * & 95,0 & 26,0 & 86,0 & 87,0 & 83,0 \\
\hline Gasto com saúde per capita (em US\$) ** & 82,3 & 34,8 & 50,5 & 53,1 & 66,3 \\
\hline 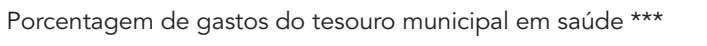 & 9,0 & 9,8 & 6,9 & 6,6 & 10,7 \\
\hline Coeficiente de mortalidade infantil/mil (< 1 ano) \# & 20,3 & 18,5 & 33,6 & 18,2 & 41,8 \\
\hline Mortalidade infantil proporcional por causas mal definidas \# & 2,9 & 37,5 & 11,7 & 26,2 & 28,0 \\
\hline População alfabetizada (\%) * & 72,2 & 43,0 & 65,7 & 64,9 & 57,7 \\
\hline População com renda insuficiente (\%) * & 52,0 & 79,9 & 59,5 & 56,7 & 70,2 \\
\hline Domicílio com canalização interna de água (\%) ** & 63,2 & 29,8 & 59,9 & 55,7 & 48,8 \\
\hline Domicílio sem instalação sanitária (\%) * & 17,6 & 58,7 & 21,6 & 29,3 & 39,9 \\
\hline Cobertura do Programa de Agentes Comunitários de Saúde \#\# & 67,0 & 81,1 & 65,4 & 65,4 & 37,0 \\
\hline Cobertura do Programa Saúde da Família \#\# & 12,9 & Início em 2001 & 12,0 & Não implantado & 17,3 \\
\hline Gestão com continuidade administrativa \#\#\# & Sim & Sim & Sim & Sim & Sim \\
\hline
\end{tabular}

Fonte: Departamento de Informação e Informática do SUS 32. Os dados de população foram aproximados visando a não identificação dos municípios.

* Censo 199133

** Gasto per capita com saúde: total gastos/população total daquele ano;

*** Proporção (\%) dos gastos do tesouro com saúde = total gastos municipais/receita total do município x 100;

\# Em 1999;

\#\# Em 2000;

\#\#\# Entrevista com gestores.

Figura 2

Grau de implantação (\%) segundo dimensões da gestão descentralizada do Sistema Único de Saúde (SUS) em cinco municípios do Estado da Bahia, Brasil, 2001.

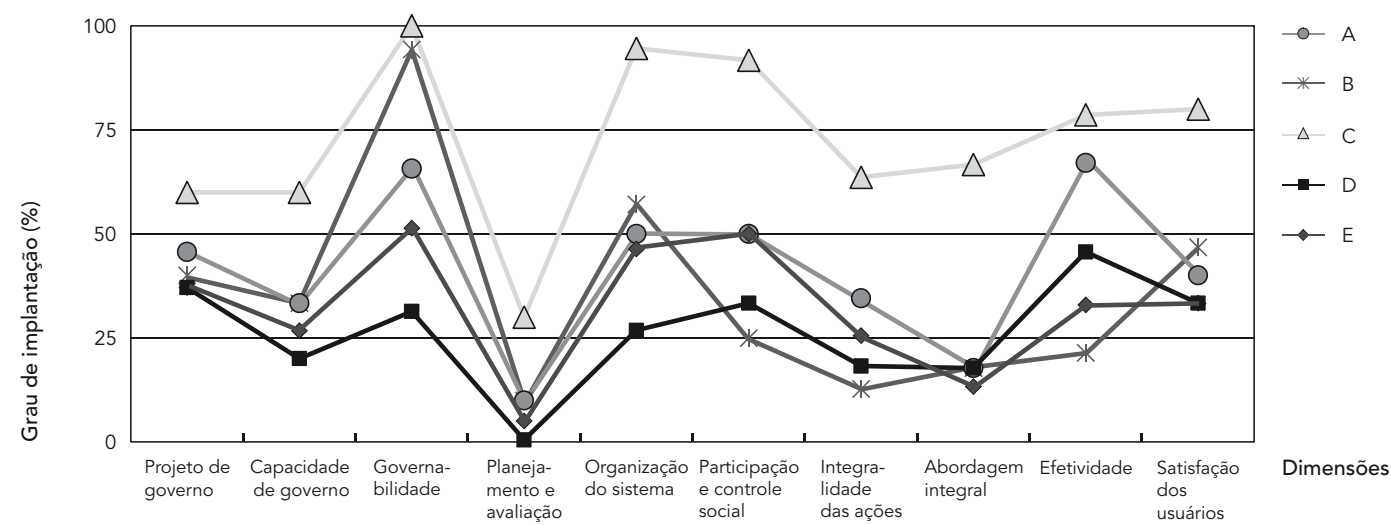


pios A, B e C havia referências aos envolvimentos de outros atores nesse processo, bem como uma maior regularidade na sua realização, embora em todos verificou-se ausência de pessoas especificamente designadas e qualificadas para o desenvolvimento dessa atividade.

A avaliação também era feita de forma assistemática não havendo instâncias ou técnicos designados para tal em todos os municípios. No Município C observou-se que havia um conhecimento acerca da relevância do planejamento e avaliação, sobre os sistemas de informação e suas possibilidades, bem como sobre as técnicas de planejamento. Porém, os entrevistados, freqüentemente referiam não haver tempo nem pessoas para o desenvolvimento dessas atividades. No Município A havia uma gerência de controle e avaliação ainda em fase de implantação. A avaliação era regular, porém voltada para a aferição da produtividade dos profissionais e cobertura assistencial.

A cobertura assistencial, no que se refere à produção ambulatorial, aumentou consideravelmente em todos os municípios estudados entre 1994 e 2001. Já a organização das práticas foi incipiente em quatro municípios devido principalmente à não existência de ações de promoção de saúde, práticas multiprofissionais e vigilância de riscos. No Município C essa dimensão foi intermediária em decorrência da existência de ações voltadas para a promoção da saúde, ainda que localizadas, estímulo à participação popular, articulação entre os níveis do sistema, utilização de protocolos e acolhimento, entre outras. Há também referências, nas entrevistas, de melhoria do acesso à alta e média complexidade, principalmente no Município C onde foi implantada uma central de marcação de consultas.

Não houve também, consistência entre os escores atingidos pelas dimensões "organização" e "resultados" das práticas (Tabela 2). Isso pode ser ilustrado nos Municípios C e D, onde a organização das práticas obteve pontuações de $65 \%$ e $18 \%$, respectivamente, e a efetividade sobre a saúde da população a partir de alguns agravos traçadores manteve-se num patamar avançado no Município C $(78,6 \%)$ e intermediário no $\mathrm{Mu}$ nicípio D (45,7\%). O Município C reduziu a internação hospitalar mais que no seu estrato de referência para todos os agravos traçadores, exceto tuberculose, enquanto o Município D reduziu a internação para diarréia e infecção respiratória aguda em menores de um ano, podendo essa redução estar relacionada com a intervenção do PACS no referido município.

Outra medida de resultados utilizada foi a $s a$ tisfação dos usuários, e essa foi consistente em relação a melhoria na organização das práticas.
No Município B, no entanto, apesar de as práticas assistenciais estarem num patamar insuficiente, houve uma razoável satisfação dos usuários com o sistema de saúde (Tabela 2). As diferenças sócio-culturais da população, eminentemente rural e as características relacionadas à história anterior de pouco acesso aos serviços de saúde no Município B, podem ter contribuído para esse resultado.

A efetividade das práticas sobre o estado de saúde da população, aferida por meio da evolução das séries de mortalidade evitável e sobre as internações, revelou concordância nos dois Municípios C e E e resultados divergentes nos Municípios A, B e D, onde a um escore global intermediário correspondeu uma efetividade avançada no Município A e efetividade incipiente no Município B. Já no Município D, a um escore global incipiente correspondeu uma efetividade intermediária.

\section{Discussão}

Os casos analisados na presente investigação, reuniram informações que permitem relacionar apenas parcialmente a descentralização da gestão da saúde com a reorganização das práticas assistenciais. O fato de todos os cinco casos encontrarem-se na gestão plena do sistema 12 , não implicou melhorias automáticas na gestão, na organização das práticas e nos seus efeitos. Tanto que dois deles foram classificados como em estágio "incipiente" em relação à "imagem-objetivo" delineada. Ou seja, a descentralização em si não pareceu capaz de assegurar mudanças nos sistemas locais de saúde, tal como foi verificado por Atkinson \& Haran 18.

A concordância encontrada entre as características de governo e a gestão da saúde revela o papel dos agentes responsáveis pela direção, tanto do governo municipal como da saúde, bem como a necessidade de um "triângulo de governo" articulado para que as condições favoráveis propiciadas pelo processo de descentralização resultem em modificações da gestão da saúde. Em particular, cabe destacar o papel da liderança do gestor municipal da saúde como fator aglutinador e mobilizador da incorporação de inovações na gestão, presentes particularmente nos Municípios C e B. O critério complementar analisado, aquele que procurava indagar acerca da adesão dos atores, revelou que o reconhecimento por parte dos profissionais dessa liderança foi fundamental para a sua eficácia. Mais que isso, o reconhecimento do projeto do governo como "seu" e a percepção de realização profissional no setor público foram elementos que compuseram 
o quadro de maior governabilidade no município mais avançado, no caso, o C.

Dois critérios utilizados que foram classificados como incipientes em todos os municípios, a saber o planejamento/avaliação e a política de recursos humanos, merecem uma exploração específica em estudos adicionais. É possível que a "imagem-objetivo" proposta para os mesmos tenha sido excessivamente idealizada. Por outro lado, a ausência de pessoas ou instâncias especificamente designadas para o desempenho dessas funções, no caso do planejamento e avaliação, revela a sua subestimação por parte dos gestores.

O fato de os gastos públicos, per capita, em saúde, não terem mostrado concordância com a organização do setor saúde e as práticas desenvolvidas, apresentando algumas vezes relações paradoxalmente inversas, revela que a análise em profundidade e desagregada para o nível do município, pode levar a conclusões distintas daquelas produzidas pelos trabalhos com dados agregados. Viana et al. 17, por exemplo, apontaram que os grupos de municípios em gestão plena menos resolutivos foram aqueles com menor disponibilidade financeira e menor capacidade de gasto com pessoal.

Os resultados pouco consistentes entre o escore global e a efetividade das práticas decorre, possivelmente, da complexidade dos determinantes da saúde nas populações. No caso específico dos Municípios A e D, os escores superiores aos esperados decorreram principalmente da redução das taxas de mortalidade por diarréia e infecção respiratória aguda naqueles municípios. Esse fato, tanto pode ser atribuído a componentes extra-setoriais como o saneamento e as condições de vida que estejam influenciando na evolução da taxa de mortalidade por esses agravos, como pode decorrer da incorporação prévia pelos serviços de medidas de controle daqueles agravos cujas ações programáticas já existem há cerca de vinte anos, a saber, o controle da diarréia e das infecções respiratórias infantis.

Houve uma consistência entre o modelo teórico elaborado e os achados empíricos. Tendo em vista que todos os municípios estudados estavam habilitados na gestão plena do sistema, possuindo características sociais semelhantes (Tabela 3), as variações observadas nos efeitos podem ser atribuídas às características de governo e da gestão, ou no sentido de Denis \& Champagne 31, ao contexto de implantação. A análise da influência da interação entre a intervenção e o contexto de implantação é importante para explicar as variações dos efeitos observados. Considerando-se a descentralização como a intervenção, o contexto como os componentes de governo ou a gestão na saúde e os efeitos, as modificações nas práticas assistenciais, de fato, os fatores contextuais (componentes do governo e gestão da saúde) podem ter contribuído para aumentar os efeitos produzidos pela intervenção no Município C (interação sinérgica) ou bloqueá-los (interação antagônica) como ocorreu nos Municípios D e E ou, ainda, sem produzir grandes influências nas práticas (interação estática) como no Município B.

Portanto, a análise da implantação da descentralização da gestão do SUS em cinco municípios da Bahia, considerados como "casos exemplares", revelou limites e possibilidades desse processo. Embora a relação entre a descentralização formal, caracterizada pela habilitação na gestão plena do sistema e o estágio de reorganização do sistema municipal aferido por meio da comparação com uma "imagem-objetivo” delineada não tenha sido verificada, cabe destacar a influência do processo normalizador federal em relação às práticas. Em alguns municípios, a ampliação dos gastos com saúde bem como a adoção de diversas medidas relacionadas com a reorganização da atenção decorreram mais de respostas a iniciativas nacionais do que da ação local. Se isso por um lado é positivo, pois mostra a importância da definição de políticas por parte do nível central na condução das ações locais, por outro, pode significar a limitação da capacidade propositiva e definidora da gestão municipal ou sua insuficiência técnica. Foram constatados múltiplos aspectos positivos e diversas repercussões do referido processo em relação às características da atenção à saúde. Verificou-se uma ampliação da cobertura assistencial em relação a diversas ações básicas de saúde em todos os municípios estudados, confirmando os resultados encontrados por Viana et al. 17. A ampliação do acesso aos serviços de alta e média complexidade foi facilitada pelo processo de descentralização na modalidade de gestão plena do sistema, reiterando os achados de Heimann 16. Ainda que a descentralização tenha sido considerada por alguns autores como parte de uma estratégia para mudar o modelo assistencial no Brasil, fortemente centrado na oferta de serviços curativos, para um outro que priorizasse o controle de riscos e causas, a promoção e prevenção e as ações intersetoriais 8, os estudos mencionados ainda não produziram evidências nessa direção.

Por outro lado, cabe um destaque ao papel desempenhado pelas características do governo sobre o estágio da reorganização alcançado pelo Sistema Municipal de Saúde. A capacidade de governo, vinculada ao perfil do gestor e dos quadros técnicos, pode ter contribuído para um melhor 
desempenho no que diz respeito às práticas assistenciais. Na governabilidade, a contribuição da subdimensão adesão dos atores, parece ter possibilitado uma melhoria nas práticas assistenciais, o que, contudo, merece aprofundamento em estudos subseqüentes.

Os problemas e insuficiências encontrados na gestão do sistema revelam essa área como crítica para futuras intervenções, principalmente no que diz respeito à institucionalização do planejamento e avaliação. Esses problemas requerem uma redefinição das atuais estratégias nacionais e regionais de intervenção, além dos aspectos relacionados à necessidade de se incentivar o desenvolvimento de projetos intersetoriais.

No que concerne à organização das práticas assistenciais, constata-se que o estágio incipiente dominou, exceção do Município C, classificado como "intermediário". Em outras palavras, as melhorias relacionadas com o acesso, acolhimento e a qualidade das práticas oferecidas à população, ainda estão muito distantes da "imagem-objetivo" delineada, sugerindo que o SUS ainda tem muito o que avançar no cuidado das pessoas. A esse respeito, as possibilidades de mudança estão mais relacionadas às características da situação municipal do que às iniciativas federais, pois diversas intervenções podem ser feitas no âmbito local visando a suprir as carências identificadas. Entre as áreas críticas na Bahia, destacam-se a gestão do sistema, a acessibilidade e a organização das práticas assistenciais no nível local. Persistem, também, problemas nos sistemas de informação que requerem intervenção principalmente no que diz respeito à investigação dos óbitos maternos e infantis evitáveis.

A inconsistência verificada em relação a alguns critérios, como no item "política de recursos humanos" que foi mal avaliado em todos os municípios, requer uma redefinição conceitual do mesmo. Possivelmente, a ampliação dos critérios relacionados a esse item, com a inclusão de aspectos relacionados ao processo de trabalho e às relações no trabalho, poderá aperfeiçoar essa avaliação em estudos posteriores.

A matriz utilizada para a avaliação não deve ser considerada como um instrumento rígido. Ao contrário, permite sucessivas redefinições, a depender do objeto e situação concreta a serem avaliados, para dar conta das especificidades de cada realidade. A sua incorporação como tecnologia para a avaliação de sistemas locais de saúde pode ser feita a partir da manutenção de critérios comuns de elevado consenso para fins de comparação entre municípios.

Com base nos resultados deste estudo algumas recomendações poderiam ser cogitadas para o aperfeiçoamento do SUS no nível local:

1) A avaliação de sistemas municipais de saúde deve ser institucionalizada nos âmbitos nacional, regional e local para subsidiar o processo decisório e otimizar a utilização dos atuais sistemas de informação; e

2) A matriz de dimensões aqui desenvolvida pode ser utilizada como um instrumento de acompanhamento do processo de descentralização nos planos nacional, regional e local.

Não obstante a constatação de que a criação de características favoráveis para o desenvolvimento e implementação adequada das políticas de saúde, no âmbito local, relacionam-se com fatores situados fora da esfera técnica, a saber, no governo e na governabilidade existem diversas possibilidades de interferência nesse processo. A ampliação da capacidade de governo através da formação de quadros estratégicos, bem como uma maior institucionalização do planejamento e da avaliação dos sistemas locais de saúde poderão contribuir para com a superação das lacunas identificadas no presente estudo.

\section{Resumo}

A compreensão das repercussões do processo de descentralização da saúde no Brasil requer a realização de investigações com enfoques diferenciados, visando a captar a complexidade do objeto a partir de distintos ângulos. Assim, o presente estudo teve por objetivo estimar o grau de implantação da gestão descentralizada da saúde, discutir a influência das características de governo e da gestão em relação ao grau de reorganização das práticas e seus efeitos na saúde da população, por meio de cinco estudos de caso em municípios selecionados da Bahia, Brasil. Foi elaborado um modelo teórico-lógico, correspondente à definição de uma "imagem-objetivo" da descentralização da saúde que orientou a elaboração de matriz de indicadores. O estudo revelou que a descentralização sozinha não explica o estágio de organização do sistema municipal de saúde e que características do governo revelaramse importantes para a reorganização das práticas. Os principais problemas encontrados localizavam-se na gestão do sistema e nas práticas assistenciais, revelando serem estas áreas críticas para futuras intervenções, principalmente no que diz respeito à institucionalização do planejamento e da avaliação, bem como em relação aos projetos intersetoriais.

Sistemas de Saúde; Gestão em Saúde; Descentralização 


\section{Colaboradores}

L. M. Vieira-da-Silva participou da formulação da pergunta, concepção do desenho da investigação, análise dos dados e redação do artigo. Z. M. A. Hartz contribui na formulação da pergunta e concepção do desenho da investigação, e participou na definição de instrumentos para coleta, na análise dos dados e redação. S. C. L. Chaves colaborou na definição de instrumentos para coleta, na análise dos dados e redação, coordenação da coleta de dados e consolidação dos mesmos. G. A. P. Silva contribuiu na definição de instrumentos para coleta, na análise dos dados, participação da coleta dos dados e consolidação dos mesmos. J. S. Paim colaborou na concepção do desenho, na definição de instrumentos para coleta e redação.

\section{Referências}

1. Khaleghian P. Decentralization and public services: the case of immunization. Soc Sci Med 2004; 59:163-83.

2. Huff-Rouselle M. Myths and realities about decentralization of health systems. Int J Health Plann Manage 2001; 16:172-4.

3. Frankish CJ, Kwan B, Ratner PA, Higgins JW, Larsen C. Social and political factors influencing the functioning of regional health boards in British Columbia (Canada). Health Policy 2002; 61:125-51.

4. Hupe LP. Implementing a meta-policy: the case of decentralisation in the Netherlands. Policy Polit 1990; 18:181-91.

5. Guimarães MCL. Descentralização da saúde, interesses e conflitos decisórios: o processo de decisão nas instâncias colegiadas estaduais, Bahia, 19931998 [Tese de Doutorado]. Salvador: Escola de Administração, Universidade Federal da Bahia; 2000.

6. Paim JS. Saúde, política e reforma sanitária. Salvador: Centro de Estudos Projetos de Saúde, Instituto de Saúde Coletiva; 2002.

7. Atkinson S, Fernandes L, Caprara A, Gideon J. Prevention and promotion in decentralized rural health systems: a comparative study from northeast Brazil. Health Policy Plan 2005; 20:69-79.

8. Paim JS. Reforma sanitária e os modelos assistenciais. In: Rouquayrol MZ, Almeida Filho N, organizadores. Epidemiologia \& saúde. Rio de Janeiro: Editora Medsi; 2003. p. 455-66.

9. Brasil. Constituição da República Federativa do Brasil. Diário Oficial da União 1988; 5 out.

\section{Agradecimentos}

Os autores agradecem a Francisco Pacheco de Souza e a Rosana Lopes Machado pelas contribuições na coleta de dados.
10. Brasil. Lei n. 8.080, de 19 de setembro de 1990. Dispõe sobre as condições para a promoção, proteção e recuperação da saúde, a organização e o funcionamento dos serviços correspondentes e dá outras providências. Diário Oficial da União 1990; 19 set.

11. Brasil. Lei n. 8.142, de 28 de dezembro de 1990. Dispõe sobre a participação da comunidade na gestão do Sistema Único de Saúde (SUS) e sobre as transferências intergovernamentais de recursos financeiros na área da saúde e dá outras providências. Diário Oficial da União 1990; 28 dez.

12. Brasil. Norma Operacional Básica do Sistema Único de Saúde/NOB-SUS 96. Brasília: Ministério da Saúde; 1997.

13. Brasil. Portaria MS/GM n. 95. Regionalização da assistência à saúde: aprofundando a descentralização com eqüidade no acesso. Norma Operacional da Assistência à Saúde NOAS-SUS 01/01. Diário Oficial da União 2001; 29 jan.

14. Vieira-da-Silva LM. Camaçari. In: Almeida CM, organizador. Os atalhos da mudança na saúde do Brasil. Serviços em nível local: uma análise comparativa (nove estudos de casos). 1a Ed. Rio de Janeiro: Organização Pan-Americana da Saúde/Organização Mundial da Saúde; 1989. p. 19-27.

15. Vieira-da-Silva LM. A descentralização das ações de saúde no município: o caso de Camaçari [Tese de Doutorado]. São Paulo: Departamento de Medicina Preventiva, Universidade de São Paulo; 1990. 
16. Heimann LS, Cortizo CT, Castro IEN, Kayano J, Rocha JL, Nascimento PR, et al. Descentralização do Sistema Único de Saúde: trilhando a autonomia municipal. São Paulo: Sociedade Brasileira de Vigilância de Medicamentos; 2000.

17. Viana ALd'A, Heimann LS, Lima LD, Oliveira RG, Rodrigues SH. Mudanças significativas no processo de descentralização do sistema de saúde no Brasil. Cad Saúde Pública 2002; 18 Suppl:139-51.

18. Atkinson S, Haran D. Back to basics: does decentralization improve health system performance? Evidence from Ceará in north-east Brazil. Bull World Health Organ 2004; 82:822-7.

19. Chen H. Theory - driven evaluations. Newbury Park: Sage Publications; 1990

20. Vieira-da-Silva LM. Avaliação do processo de descentralização das ações de saúde. Ciênc Saúde Coletiva 1999; 4:331-9.

21. Yin RK. Case study designs for evaluating high-risk youth programs: the program dictates the design, in applications of case study research. In: Yin RK, editor. Applications of case study research. Newbury Park: Sage Publications; 1993. p. 77-93.

22. Vieira-da-Silva LM, Hartz ZMA, Chaves SCL, Silva GAP. Metodologia para análise da implantação de processos relacionados à descentralização da atenção à saúde no Brasil. In: Hartz ZMA, Vieirada-Silva LM, organizadores. Avaliação em saúde: dos modelos teóricos à prática na avaliação de programas e sistemas de saúde. Rio de Janeiro: Editora Fiocruz/Salvador: EDUFBA; 2005. p. 207-53.

23. Mercer SL, Goel A. Program evaluation in the absence of goals: a comprehensive approach to the evaluation of a population-based breast cancer screening program. Canadian Journal of Program Evaluation 1994; (9):97-112.

24. Medina MG, Silva GAP, Aquino R, Hartz ZMA. Uso de modelos teóricos na avaliação em saúde: aspectos conceituais e operacionais. In: Hartz ZMA, Vieira-da-Silva LM, organizadores. Avaliação em saúde: dos modelos teóricos à prática na avaliação de programas e sistemas de saúde. Rio de Janeiro: Editora Fiocruz/Salvador: EDUFBA; 2005. p. 41-63.
25. Matus C. Política, planejamento e governo. 3a Ed. v. I. Brasília: Instituto de Pesquisa Econômica Aplicada; 1993.

26. Donabedian A. Methods of deriving the criteria, in explorations in quality assessment and monitoring. Michigan: H. A. Press Editor; 1982.

27. Donabedian A. The seven pillars of quality. Arch Pathol Lab Med 1990; 114:1115-8.

28. Kessner M, Kalk CE, Singer J. Assessing healthy quality: the case for tracers. N Engl J Med 1973; 288:189-94.

29. Souza LEPF, Vieira-da-Silva LM, Hartz ZMA. Conferência de consenso sobre a imagem-objetivo da descentralização da atenção à saúde no Brasil. In: Hartz ZMA, Vieira-da-Silva LM, organizadores. Avaliação em saúde: dos modelos teóricos à prática na avaliação de programas e sistemas de saúde. Rio de Janeiro: Editora Fiocruz/Salvador: EDUFBA; 2005. p. 65-102.

30. Ferreira JR. El analisis prospectivo de la educación médica en América Latina. Educ Méd Salud 1988; 22:242-367.

31. Denis JL, Champagne F. Análise da implantação de programas. In: Hartz ZMA, organizadores. Avaliação em saúde: dos modelos conceituais à prática na análise da implantação de programas. Rio de Janeiro: Editora Fiocruz/Salvador: EDUFBA; 2005. p. 49-88.

32. Departamento de Informação e Informática do SUS/Secretaria Executiva do Ministério da Saúde. Informações em saúde. http://www.datasus.gov. br/datasus/datasus.php (acessado em Set/2001).

33. Instituto Brasileiro de Geografia e Estatística. Censos demográficos e contagem populacional para os anos intercensitários, estimativas preliminares dos totais populacionais, estratificadas por idade e sexo pelo MS/SE/Datasus. http://www.datasus. gov.br/datasus/datasus.php?area=359A1B379C6 D0E0F359G23H0I1Jd6L26M0N\&VInclude $=. . /$ site/ infsaude.php (acessado em Set/2001).

Recebido em 05/Ago/2005

Versão final reapresentada em 05/Mai/2006

Aprovado em 20/Jun/2006 